\title{
Qualitative Analysis of Grapevine Tannins by Mass Spectrometry and their Inhibitory Effect on Stilbene Oxidase of Botrytis cinerea
}

\author{
Camille Perret ${ }^{\mathrm{a}}$, Roger Pezet ${ }^{\mathrm{b}}$, and Raffaele Tabacchi ${ }^{\mathrm{a} *}$
}

\begin{abstract}
The pure tannin extracts from grape berries of Gamay and Gamaret varieties contain a mixture of proanthocyanidins and proanthocyanidin gallates. A sensitive LC-MS method has been developed for the qualitative analysis of the oligomers and polymeric fractions. The potential of ESI-IT or MALDI-TOF mass spectrometry permit us to characterize these tannins. Polymers were fractionated according to the mean degree of polymerization (mDP), using either a divinylbenzene phase or a glass powder. The mDP is obtained by thiolysis reaction and the fractions are analyzed by MS. Polymeric proanthocyanidins (PPRA) were purified from grape berries at full bloom, pea size, bunch closure, véraison and harvest, their mDP calculated and their inhibitory capacity on $B$. cinerea stilbene oxidase tested at 3.5 to $70 \mathrm{nmoles}$ for $3 \mathrm{ml}$ of enzymatic reaction volume. ED50 of PPRA concentrations was measured for each developmental stage and for both grape varieties Gamay (sensitive to grey mould) and Gamaret (resistant). The higher the mDP of PPRA fraction, the more effective is the inhibitory activity. From grape berry pea size, mDP of PPRA of Gamaret (9.53 to 11.77) were always higher than those of Gamay (7.46 to 8.74) and ED50 of Gamaret PPrA were under 5 nmoles $3 \mathrm{ml}^{-1}$ while ED50 of Gamay PPRA were at 6.5 to 7.25 nmoles $3 \mathrm{ml}^{-1}$.
\end{abstract}

Keywords: Botrytis cinerea · Grape berries · Inhibition of stilbene oxidase · Mass spectrometry Polymeric tannins - Vitis vinifera

\section{Introduction}

Gray mould, caused by Botrytis cinerea, is one of the most important diseases of grapes. Flowering clusters can be infected by $B$. cinerea, but it does not develop in the berries until they ripen [1]. This quiescent stage is probably maintained by an unfavorable environment [2].

Resveratrol (1) and pterostilbene (2) are present in immature berries acting as phytoalexins. B. cinerea produces a hydroxystilbene-degrading enzyme, identified as a

\footnotetext{
${ }^{\star}$ Correspondence: Prof. R. Tabacchi alnstitut de Chimie, Université de Neuchâtel 51, Avenue de Bellevaux CH-2007 Neuchatel

Tel.: +4132718 2429

Fax: +41327182511

E-Mail: raphael.tabacchi@unine.ch

bStation fédérale de recherches en production végétale de Changins

Route de Duillier

$\mathrm{CH}-1260$ Nyon
}

laccase-like stilbene oxidase. In a previous work we described the structure and the concentration of constitutive berry phenolic compounds from Gamay and Gamaret varieties and their biological activities [3].

At low concentration polymeric tannins of the healthy grape berries of Gamaret and Gamay cause a rapid inhibition of this laccase. The pure tannin extract contains a mixture of proanthocyanidins and galloylated proanthocyanidins 3. These polar compounds are difficult to isolate and characterize due to their high molecular weight and their polarity.

If phytoalexins are important factors in the resistance of a plant to phytopathogenic fungi, the ability of a parasite to detoxify these compounds could be a major component of the mechanisms of pathogenicity of parasites. Botrytis cinerea produces an hydroxystilbene-oxidizing enzyme [1], belonging to the laccase family, which is able to oxidize into dimers both pterostilbene and resveratrol, known grapevine phytoalexins [4]. Pterostilbene, the most fungi- toxic stilbene, is detoxified when oxidized. Resveratrol, which is slightly toxic, is converted into the dehydrodimer, which is a slightly more potent antifungal agent [5]. This $B$. cinerea stilbene oxidase is inhibited by the tannins contained in the grape berries [6]. The aim of this study is to define which tannins are responsible for this enzyme inhibition.

\section{Results}

The pure tannin extracts from grape berries of Gamay (sensitive to Botrytis) and Gamaret (resistant) varieties containing a mixture of proanthocyanidins and proanthocyanidin gallates have been analyzed.

Electrospray-mass spectrometry combined with sequential tandem MS (ESIMS/MSn) is a successful analytical tool providing molecular mass and structural information of this polyphenol mixture. Sensitive detection is obtained in negative ion mode by any buffer addition, generating an 

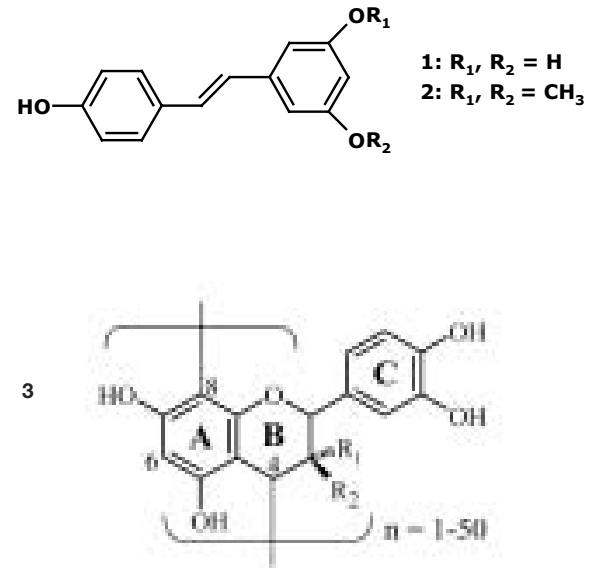

Catechine: $\mathrm{R}_{1}-\mathrm{H}, \mathrm{H}-\mathrm{OH}$ Epicatechin: $R_{1}=0 H, R_{2}=H$
Epicatechin-3-0-Eallate: $R_{2}=H_{1} R_{2}-0$ - $C$

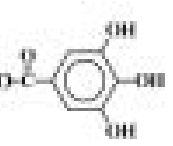

This method has been developed to test the enzyme's inhibition by tannins in relation to their molecular weight. The collected fractions have an average mDP that varies between 2.12 and 17.69 (see Fig. 2) in an increasing order, corresponding to molecular weights between 290 to $~ 5200$.

The fractions were analyzed by ESI [6], MALDI-TOF, or MALDI - FT-ICR-MS. MALDI-TOF/MS is a suitable method to analyze these fractions [9][10]. The use of a DHB matrix increases sensitivity and high molecular weight ions are also strongly extracted and detected in the positive ion mode.

Fig. 3 shows the spectra of fractions $1-8$. Fractions 9 and 10 could not be ionized due to the high mDPs (17.00 and 17.69). Ions are detected with a sodium adduct $[\mathrm{M}+\mathrm{Na}]^{+}$. Proanthocyanidins, galloylated and polygalloylated proanthocyanidins can be observed on these spectra with a mass range up to $4000 \mathrm{amu}$. The highest molecular weight clearly detected is the digalloylated decamer at $\mathrm{m} / z, 3211$. We should point out that the detection of high molecular weight proanthocyanidins is less sensitive than the detection of low molecular weight species. Therefore a shift in the high mass range detection is weakly observed in the fractions 6 to 8 . The loss of sensitivity may come from fragmentations during time of flight or as ions are extracted from the matrix. Low masses from the matrix might saturate the detector, preventing the observation of higher polymers. In this case ion intensities are not related to their concentration in the sample.

Polymeric proanthocyanidins (PPRA) were purified from grape berries at full bloom, pea size, bunch closure, véraison and harvest, their mDP calculated and their inhibitory capacity on B. cinerea stilbene oxidase tested at 3.5 to 70 nmoles for $3 \mathrm{ml}$ intense deprotonated molecular ion. The mass range of these polymers is between 290 (catechin) and 3100u (decamers of proanthocyanidins). A quasimolecular ion at $\mathrm{m} / \mathrm{z} 255[\mathrm{M}-\mathrm{H}]^{-}$corresponding to the molecular weight of pterostilbene, is present in these active extracts. This information induces the possibility of tannin-complex formation or ester bond linkage to prevent stilbene degradation by the laccase. A sensitive LC-MS method has been developed in our laboratory for the qualitative analysis of the oligomers. The potential of an IT/MS (ion trap) equipped with ESI (electrospray) interface permit us to induce multiple stage fragmentation (MSn), making the characterization of these compounds easier [7].

Different methods of tannin fractionation and analysis have been developed. Oligomeric tannins have been isolated with exclusion gels. The polymeric fraction contains a mixture of proanthocyanidins and galloylated proanthocyanidins of high molecular weight. The acid-catalyzed degradation (thiolysis) in the presence of a nucleophile reagent (phenyl-methanethiol) was used for the determination of the mean degree of polymerization (mDP) of condensed tannins [8]. The cleavage of the carbon-carbon link releases the flavan-3-ol units without stereochemical changes. After degradation, the reaction products are analyzed by HPLC in order to determine the $\mathrm{mDP}$ of the polymeric fraction (Fig. 1).

Polymeric tannins were fractionated according to the mDP, using either a divinylbenzene phase or a glass powder with a gradient of $\mathrm{MeOH} / \mathrm{CHCl}_{3}$. The $\mathrm{mDP}$ is then calculated by means of a thiolysis reaction.

\begin{tabular}{|lccc|}
\hline \multicolumn{1}{|c}{ Cpds } & number & MW & RT \\
\hline Catechin & 1 & 290 & 7.8 \\
Epicatechin & 2 & 290 & 8.6 \\
Epicatechin-3-O-gallate & 3 & 442 & 10.2 \\
Benzylthiocat. or & 4 & 412 & 13.6 \\
Epicatechin & & & \\
Benzylthioepigallocatechin & 5 & 428 & 13.8 \\
Benzylthiocatechin & 6 & 412 & 14.8 \\
Benzylthioepicatechin & 7 & 412 & 15.2 \\
Benzylthioepicatechin-3- & 8 & 564 & 15.8 \\
O-gallate & & & \\
\hline
\end{tabular}

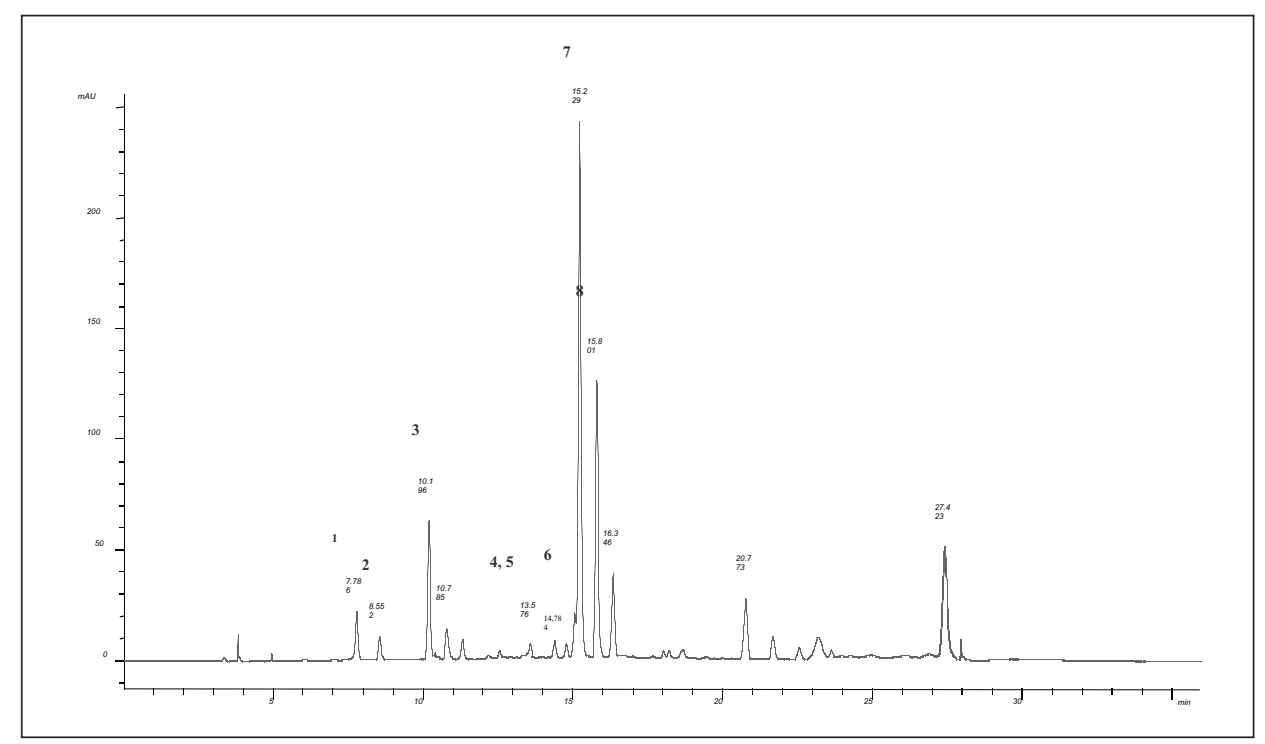

Fig. 1. HPLC chromatogram of the thiolysis reaction 


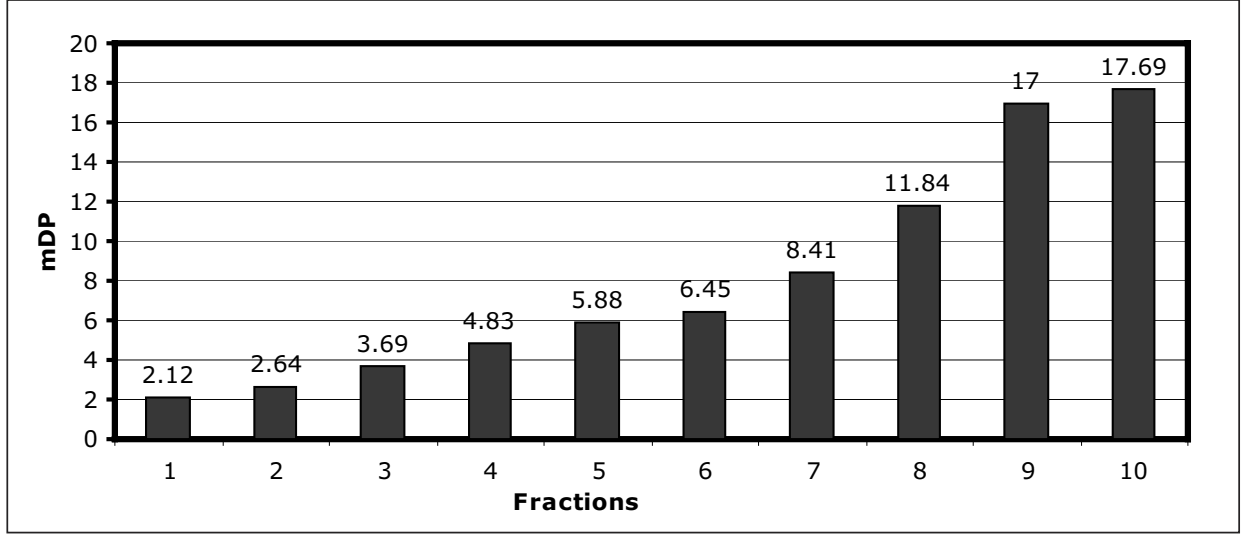

Fig. 2. Separation of a polymeric fraction according to the mDP

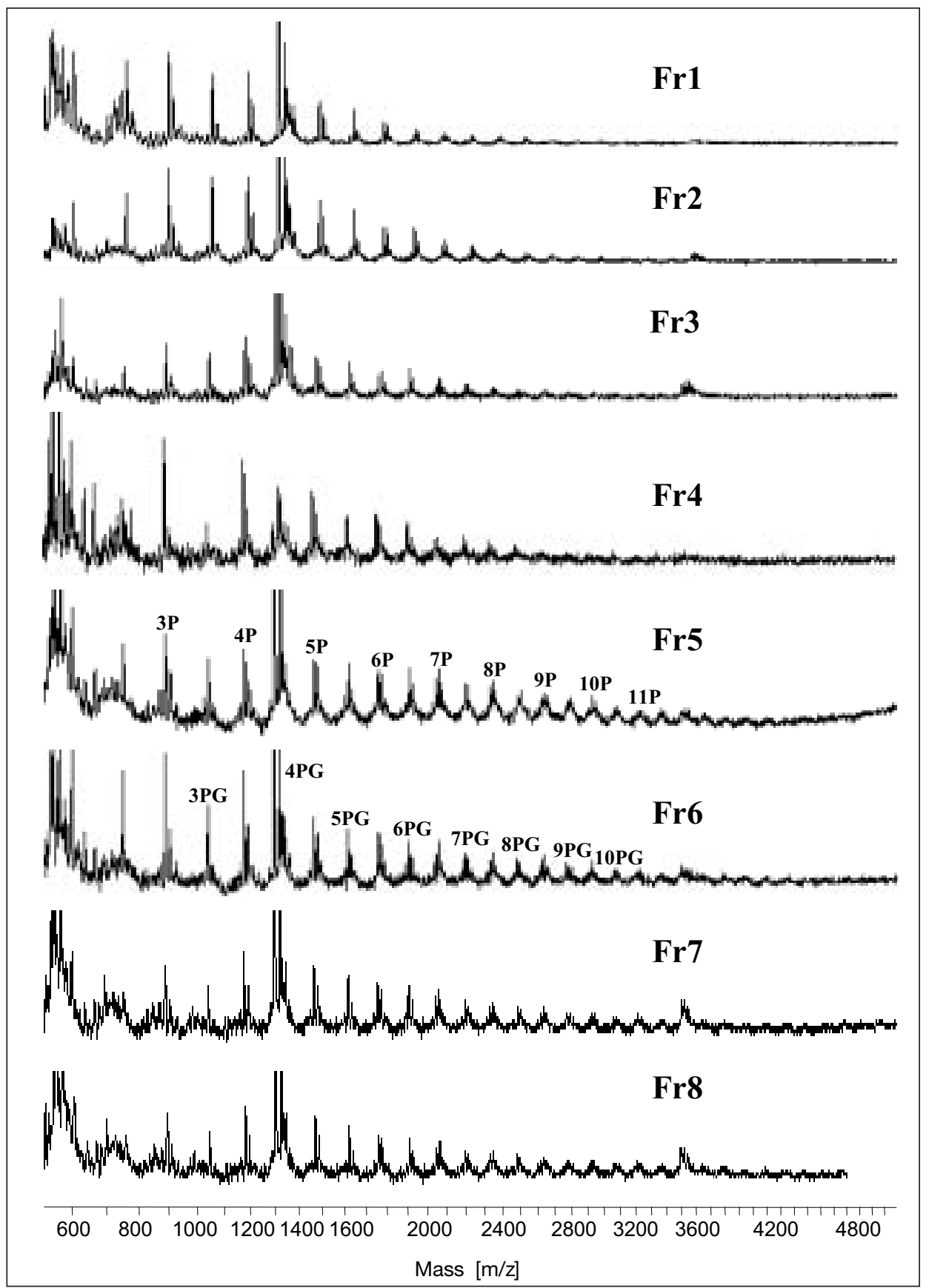

Fig. 3. MALDI-TOF/MS spectra of fractions 1 to 8 obtained by fractionation of a polymeric tannin extract on relite SP411. The calibrants show peaks at $\mathrm{m} / \mathrm{z} 1296.7$ and 3495.9. The peak with the highest intensity in each group of peaks corresponds to the non-galloylated proanthocyanidins $(P)$ or the galloylated proanthocyanidins $(P G)$. The numbers 3-10 indicate the number of units. of enzymatic reaction volume. The effective dose (ED50) of PPRA concentrations was measured for each developmental stage and for both grape varieties, Gamay and Gamaret.

The inhibitory activity of stilbene oxidase by PPRA is closely linked to their $\mathrm{mDP}$. The higher the $\mathrm{mDP}$, the more effective the enzyme inhibition. Fig. 4 shows this correlation in the case of Gamaret. From pea size to harvest, mDP of Gamaret PPRA fractions (9.73 to 11.77 ) are always higher than those of Gamay (7.46 to 8.74) (Fig. 5A). These results demonstrate that PPRA fractions of Gamaret always have a more efficient activity. This is shown by the calculation of effective doses (ED50) of PPRA which are able to decrease by $50 \%$ the activity of B. cinerea stilbene oxidase. ED50s of Gamaret PPRA were always under 5 nmoles $3 \mathrm{ml}^{-1}$ from pea size to harvest, while those of Gamay increased during this time (Fig. 5C). In addition, the concentration of PPRA fraction per gram of fresh weight of berries is twice as high in Gamaret than in Gamay at harvest (Fig 5B). Higher concentrations of a more enzyme inhibitory fraction in Gamaret berries can explain the lack of B. cinerea development in this variety. Effectively, the growth of B. cinerea and the appearance of grey mould in grape berries is linked to the inhibition of macerating enzymes excreted by the fungus [11]. The qualitative and quantitative characteristics of Gamaret PPRA can explain its resistance to grey mould and chemical analysis and $\mathrm{mDP}$ characterization of grape berry tannins is a powerful tool for the breeding of grapes for natural $B$. cinerea resistance.

\section{Experimenta}

\section{Plant Material}

Immature berries of Vitis vinifera (var. Gamay) collected at the Federal Agriculture Research Center (Switzerland) were freeze dried and ground. The powder was extracted in $\mathrm{MeOH} /$ acetone/ $\mathrm{H}_{2} \mathrm{O}$ 40:40:20 $\mathrm{v} / \mathrm{v} / \mathrm{v}$. Solvents were eliminated by evaporation under vacuum. The water fraction was then extracted three times with hexane, in order to remove chlorophyll and lipophilic compounds. The tannin fraction was deposited over a LH-20 column (Pharmacia Biochem) and eluted with $\mathrm{MeOH} / \mathrm{H}_{2} \mathrm{O} 50: 50 \mathrm{v} / \mathrm{v}$ to remove oligomeric tannins and then with acetone $/ \mathrm{H}_{2} \mathrm{O}$ 75:25 $\mathrm{v} / \mathrm{v}$. The last fraction, containing the polymeric tannins, was purified by chromatography over Toyopearl HW-40S (Tosohaas, Japan) with $\mathrm{MeOH} / \mathrm{H}_{2} \mathrm{O}$ 50:50 v/v, pure methanol and finally acetone $/ \mathrm{H}_{2} \mathrm{O} 75: 25 \mathrm{v} / \mathrm{v}$. 


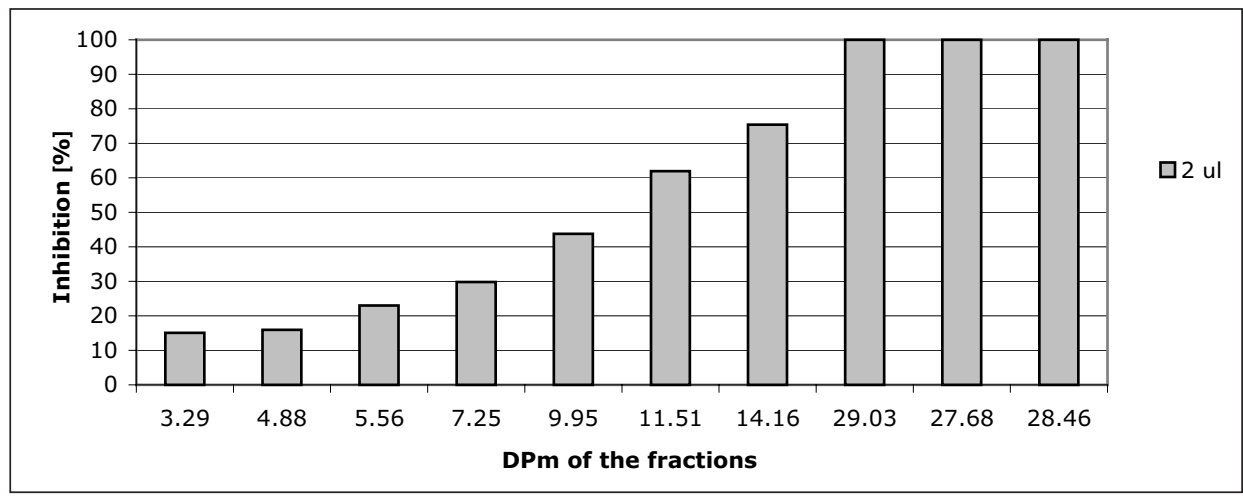

Fig. 4. Inhibition activity of the different dPM Gamaret fractions

$\mathbf{A}$

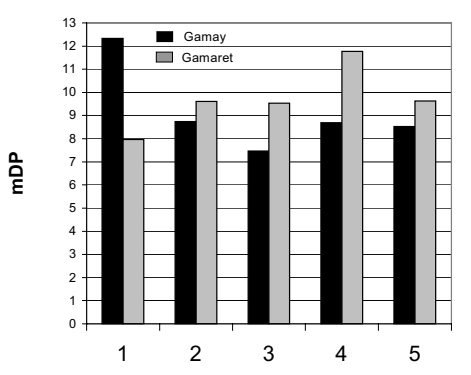

B

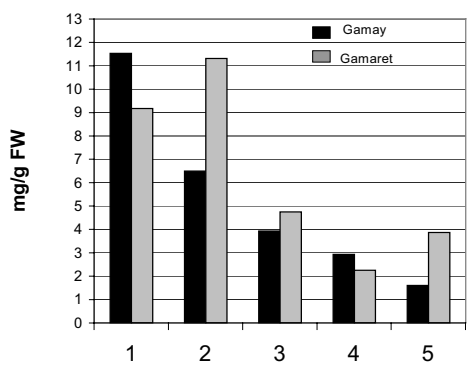

C

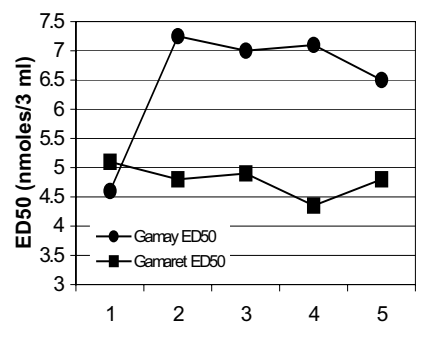

Fig. 5. Mean degree of polymerization (A), fresh weight (B) and ED50 on inhibition of stilbene oxidase activity (C) of proanthocyanidin of Gamay and Gamaret. Developmental stages of bunches; 1: full bloom, 2: pea size, 3: bunch closure, 4: vérasion, 5: harvest.

\section{Fractionation According to the $\mathrm{mDP}$}

$60 \mathrm{mg}$ of the polymeric fraction was dissolved in $1 \mathrm{ml}$ of $\mathrm{MeOH}$ and mixed with a small quantity of relite SP411 (styrene-divinylbenzene phase, Resindion, Italy). The solvent was evaporated under vacuum.

The dried mixture was deposited on the top of the relite SP411 column equilibrated with $\mathrm{CHCl}_{3} / \mathrm{MeOH} 85 \%: 15 \%$ v/v. The elution was performed as follow: starting with $30 \mathrm{ml}$ of a mixture of $\mathrm{CHCl}_{3} / \mathrm{MeOH}$ $85 \%: 15 \% \mathrm{v} / \mathrm{v}$ (fraction 1) the $\mathrm{MeOH}$ was increased in 5\% steps at each elution (30 ml) until $\mathrm{CHCl}_{3} / \mathrm{MeOH} 45 \%: 55 \% \mathrm{v} / \mathrm{v}$ (fractions 2 to 9 ) and finally with $60 \mathrm{ml}$ of pure $\mathrm{MeOH}$ (fraction 10). This experiment was performed three times, in order to determine an average $\mathrm{mDP}$.

\section{Thiolysis Reaction}

$1 \mathrm{mg}$ of the mDP fraction was dissolved in $500 \mu \mathrm{l}$ of $\mathrm{MeOH}$ (HPLC grade, SDS, France) and $50 \mu \mathrm{l}$ of this solution was transferred into a $1 \mathrm{ml}$ vial (Infochroma AG, Switzerland). $50 \mu$ of a $0.2 \mathrm{M} \mathrm{HCl}$ methanolic solution containing 5\% of phenyl-methanethiol (Fluka, Switzerland) was added. The vial was crimped and heated 2 min at $90{ }^{\circ} \mathrm{C}$.

$20 \mu \mathrm{l}$ of the reaction solution was injected onto a RP-C ${ }_{18}$ column (Nucleosil-100, $7 \mu \mathrm{m}$, 250-4.6, Bischoff, Switzerland) equipped with an $\mathrm{RP}-\mathrm{C}_{18}$ precolumn, connected to an HPLC system (HP 1050, Agilent) with an UV detector (HP 1040, Agilent) set at 280 $\mathrm{nm}$. Elution as follows: flow rate $0.8 \mathrm{ml} / \mathrm{min}$,
A: $\mathrm{H}_{2} \mathrm{O} / \mathrm{HCOOH}$ 98:2 v/v; $\mathrm{B}: \mathrm{CH}_{3} \mathrm{CN} /$ $\mathrm{H}_{2} \mathrm{O} / \mathrm{HCOOH} \quad 80: 18: 2 \mathrm{v} / \mathrm{v} / \mathrm{v} ;$ gradient $85: 15$ to $25: 75$ in $15 \mathrm{~min}$, from $25: 75$ to $100 \%$ of B in 5 min, then $100 \%$ of B for 4 min and then back to the starting conditions.

\section{ESI/MS and MALDI-TOF Analysis}

ESI/MS analyses were carried out on a LCQ from Thermofinnigan (San Jose, USA) equipped with an electrospray source interface and an ion trap. Nitrogen was used as sheath gas. One tenth of the total flow rate of the HPLC was diverted into the source and the remaining was vented. The LC/MS runs were recorded in the positive and in the negative ion mode respectively.

MALDI-TOF/MS analyses were performed on a Dynamo instrument from Thermo Bioanalysis (England) equipped with a time of flight analyzer, an UV nitrogen laser $(377 \mathrm{~nm})$ and a delay extraction. Internal calibration standards are insulin bovine B-chain oxidized at m/z 3495.9 and human angiotensine I at m/z 1296.7 (Sigma, Switzerland). The matrix (2,5-dihydroxybenzoic acid (DHB), Fluka, Switzerland) was dissolved in a mixture of $\mathrm{MeOH} / \mathrm{H}_{2} \mathrm{O}$ 4:6 v/v until saturation. Samples were solubilized in $\mathrm{MeOH}(0.01$ $\mathrm{mg} / \mu \mathrm{l})$ and $3 \mu \mathrm{l}$ of this solution were diluted in a $10 \mu \mathrm{l}$ of the matrix. $2 \mu \mathrm{l}$ of this mixture was deposited on a metal plate until crystallization. Analyses were recorded in the positive ion mode.

\section{Acknowledgements}

Financial support from the Swiss National Science Foundation (20-61879.00 and NCCR. Plant Survival) is gratefully acknowledged.

Received: July 14, 2003

[1] R. Pezet, FEMS Microbiology Letters 1968, 167, 203.

[2] R. Pezet, O. Viret, C. Perret, R. Tabacchi, J. Phytopathol. 2003, 151, 208

[3] G. Goetz. A. Fkyerat, N. Metais, M. Kunz, R. Tabacchi, R. Pezet, V. Pont, Phytochemistry 1999, 52, 759

[4] R. Pezet, V. Pont, K. Hoang-Van, Physiological and Molecular Plant Pathology 1991, 39, 441-450.

[5] A.C. Breuil, M. Adrian, N. Pirio, P. Meunier, R. Bessis, P. Jeandet, Tetrahedron Letters 1998, 39, 537.

[6] R. Pezet, V. Pont, K. Hoang-Van, in 'Recent Advances in Botrytis Research', Eds.: K. Verhoeff, N.E. Malatrakis, B. Williamson, Proceeding of the 10th International Symposium, Heraklion, Crete, Greece, 1992 p. 87.

[7] C. Perret, R. Tabacchi, R. Pezet, Eur. J. Mass. Spectrometry 2001, 7, 419.

[8] S. Matthiew, I. Mila, A. Scalbert, B. Pollet, C. Lapierre, C. Hervé du Penhoat, C. Rolando, D.M. Donnely, J. Agric. Food Chem. 1997, 45, 1195

[9] C.G. Kruger, N.C. Dopke, P.M. Treichel, J. Folts, J.D. Reed, J. Agric. Food Chem. 2000, 48, 1663

[10] C. Perret, R. Tabacchi, R. Pezet, Phytochemical Analysis 2003, in press

[11] G. Hill, F. Stellwaag-Kittler, G. Huth, E. Schlösser, Phytopath Z. 1981, 102, 328 